\title{
References
}

1 Glynn JR, Murray J, Bester A, et al. High rates of recurrence in HIV-infected and HIV-uninfected patients with tuberculosis. J Infect Dis 2010; 201: 704-711.

2 Sonnenberg P, Murray J, Glynn JR, et al. HIV-1 and recurrence, relapse, and reinfection of tuberculosis after cure: a cohort study in South African mineworkers. Lancet 2001; 358: 1687-1693.

3 Verver S, Warren RM, Beyers N, et al. Rate of reinfection tuberculosis after successful treatment is higher than rate of new tuberculosis. Am J Respir Crit Care Med 2005; 171: 1430-1435.

4 Crampin AC, Mwaungulu JN, Mwaungulu FD, et al. Recurrent TB: relapse or reinfection? The effect of HIV in a general population cohort in Malawi. AIDS 2010; 24: 417-426.

5 Marx FM, Dunbar R, Enarson DA, et al. The temporal dynamics of relapse and reinfection tuberculosis after successful treatment: a retrospective cohort study. Clin Infect Dis 2014; 58: 1676-1683.

6 Wood R, Lawn SD, Caldwell J, et al. Burden of new and recurrent tuberculosis in a major South African city stratified by age and HIV-status. PLoS One 2011; 6: e25098.

7 Ayles H, Schaap A, Nota A, et al. Prevalence of tuberculosis, HIV and respiratory symptoms in two Zambian communities: implications for tuberculosis control in the era of HIV. PLoS One 2009; 4: e5602.

8 Corbett EL, Bandason T, Cheung YB, et al. Prevalent infectious tuberculosis in Harare, Zimbabwe: burden, risk factors and implications for control. Int J Tuberc Lung Dis 2009; 13: 1231-1237.

9 den Boon S, Lill SW, Borgdorff MW, et al. High prevalence of tuberculosis in previously treated patients, Cape Town, South Africa. Emerg Infect Dis 2007; 13: 1189-1194.

10 Ayles $\mathrm{H}$, Muyoyeta $\mathrm{M}, \mathrm{Du} \mathrm{T}$, et al. Effect of household and community interventions on the burden of tuberculosis in southern Africa: the ZAMSTAR community-randomised trial. Lancet 2013; 382: 1183-1194.

11 Ayles HM, Sismanidis C, Beyers N, et al. ZAMSTAR, The Zambia South Africa TB and HIV Reduction Study: design of a $2 \times 2$ factorial community randomized trial. Trials 2008; 9: 63 .

12 Harries AD, Chimzizi RB, Nyirenda TE, et al. Preventing recurrent tuberculosis in high HIV-prevalent areas in sub-Saharan Africa: what are the options for tuberculosis control programmes? Int J Tuberc Lung Dis 2003; 7: 616-622.

13 Fitzgerald DW, Desvarieux M, Severe P, et al. Effect of post-treatment isoniazid on prevention of recurrent tuberculosis in HIV-1-infected individuals: a randomised trial. Lancet 2000; 356: 1470-1474.

14 Churchyard GJ, Fielding K, Charalambous S, et al. Efficacy of secondary isoniazid preventive therapy among HIV-infected Southern Africans: time to change policy? AIDS 2003; 17: 2063-2070.

15 Kranzer K, Afnan-Holmes H, Tomlin K, et al. The benefits to communities and individuals of screening for active tuberculosis disease: a systematic review. Int J Tuberc Lung Dis 2013; 17: 432-446.

\section{Fixed-dose combination and therapeutic drug monitoring in tuberculosis: friend or foe?}

To the Editor:

Tuberculosis (TB) remains one of the world's deadliest infectious diseases. The World Health Organization (WHO) estimated that, in 2014 alone, 9.6 million people fell ill with TB and 1.5 million died due to the disease [1]. South-East Asia and Western Pacific Regions accounted for 58\% of these [1]. As most deaths from TB can now be prevented, efforts must be accelerated to ensure the targets of the Sustainable Development Goals are reached [1].

Drug-susceptible TB is treated with first-line anti-TB drugs, consisting of 2 months of isoniazid, rifampicin, pyrazinamide and ethambutol, thereafter continued with only isoniazid and rifampicin for 4 months [2]. This treatment regimen achieves success rates of approximately $85 \%$ worldwide [1]. However, there is room for improvement as non-adherence and inappropriate prescription of TB therapy are believed to be key reasons of TB treatment failure and development of drug resistance [3]. Therefore, one of the WHO strategies to combat active TB was the introduction of fixed-dose combination (FDC) formulations. FDC tablets, containing two to four first-line anti-TB drugs, are used to simplify TB treatment and thereby increase compliance and reduce prescription errors [4]. A recent meta-analysis of 13 randomised controlled trials (RCTs), showed non-significant differences in negative treatment outcomes following treatment with FDC or single drug formulations of TB-drugs [5].

Over the last few years it has become clear that drug exposure of anti-TB drugs is of importance. A meta-analysis of 13 randomised studies showed that microbiological failure and relapse occur more frequently in rapid acetylators of isoniazid than in slow acetylators. Observed pharmacokinetic variability 
was significantly associated with therapeutic failure and acquired drug resistance [6]. Although high doses of isoniazid and rifampicin have been shown to be well tolerated [7], increasing doses of ethambutol and pyrazinamide have been shown to cause ocular- and hepatotoxicity respectively [8]. Additionally, anti-TB drug-induced liver injury is not uncommon, particularly in the Chinese population with an incidence of $13 \%$ in a recent study [9].

Therapeutic drug monitoring (TDM) is a technique that allows individual dosing based on drug plasma concentrations. The use of TDM is considered useful for an effective and well-tolerated treatment regimen [10]. Problems related to TDM, for instance costs, logistics and invasiveness, can be addressed by using new tools such as limited sampling, dried blood spots (DBS) and simultaneous analysis of all first-line anti-TB drugs $[8,10,11]$. GHIMIRE et al. [11] suggest a programmatic setting for performing TDM with these new tools, which seems feasible for global use. Even though the costs of performing TDM are estimated to be US $\$ 560$ for testing the four first-line drugs, this expense is negligible if a case of multidrug-resistant (MDR)-TB can be avoided [12].

However, the "number of patients needed to perform TDM", defined by the number of patients who need to be subjected to TDM to prevent acquired MDR-TB in one patient will determine the true effectiveness of this health-care intervention. As variability in drug exposure and acquired drug resistance can differ per setting collection of local data is important to make a well-informed decision on implementation of TDM.

One might think that implementation of TDM in programmatic treatment will end the use of FDC, since each individual drug would have to be dosed based on the drug plasma concentration. However, the opposite may be true. Therefore, the aim of this contribution is to discuss the role of TDM in further improving efficacy, safety and tolerability of FDC regimens. To illustrate our proposal, we present practical advice for TDM and FDC tablet selection in a relevant clinical setting.

Given the overall relevance and availability of information, the chosen setting to exemplify this is China.

In our strategy to combine TDM and FDC, we included first-line drugs (i), available FDC tablets (ii), choice of FDC tablets in combination with TDM (iii) and logistical considerations (iv). For this example we have made the following assumptions. i) Based on previous studies, early dose adjustment of pyrazinamide, rifampicin and isoniazid are needed in order to prevent acquired drug resistance 2 months after start of treatment and improve long-term treatment outcome [13]. ii) Current standard TB treatment for adults consists of isoniazid $5 \mathrm{mg} \cdot \mathrm{kg}^{-1}$; rifampicin $10 \mathrm{mg} \cdot \mathrm{kg}^{-1}$; pyrazinamide $25 \mathrm{mg} \cdot \mathrm{kg}^{-1}$ and ethambutol $15 \mathrm{mg} \cdot \mathrm{kg}^{-1}$, dependent on weight [2]. FDC tablets based on these doses have been developed to enable weight-banded dosing. iii) TDM is suggested only in patients with a higher risk of insufficient plasma concentrations [10], since it is neither feasible nor cost-effective to perform TDM in all patients [12]. TDM should be performed 2 weeks after start of drug-susceptible anti-TB treatment. Based on plasma concentrations, the appropriate FDC tablets can be selected. Two weeks after dose adjustment, the drug concentrations have to be confirmed. In the continuation phase, the appropriate FDC combination of rifampicin and isoniazid can be selected, based on earlier measured drug concentrations. We consider drug exposure that differs at least $25 \%$ from target concentrations as clinically relevant, in accordance with Drug regulatory agencies' guidelines. iv) Patients from rural areas in China could use dried blood spots sampling for TDM at a community health centre, to enable TDM in resource-limited settings.

The results of these assumptions have led to a practical and simple approach for FDC tablet selection and implementation of TDM in a clinical setting, exemplified in figure 1. In the case of drug exposure below the target value, the dose should be increased. The plasma concentration of the drug showing the lowest concentration is the starting point for increasing the daily dose FDC containing all four first-line anti-TB drugs. Although one may be worried about toxic concentrations of the other drugs, this is suspected to be overestimated as the percentage of the dose increase did not exceed $25 \%$. Only when pyrazinamide or ethambutol are above target value, is the dose lowered, as higher doses of isoniazid and rifampicin are considered to be safe and well tolerated [7]. Although limited evidence suggests a higher dose of pyrazinamide is safe [14], we recommend caution while waiting for the results from clinical trials. However, pyrazinamide has been found to play an important role in anti-TB treatment; therefore, it should not be ignored, but adjusted, keeping toxicity in mind [13]. Additionally, follow-up measurements after dose adjustment assure that dose interventions are successful and too high drug exposure will not go unnoticed. The role of ethambutol in first-line anti-TB treatment is still up for debate, however the WHO no longer recommends omission of ethambutol and therefore it was incorporated in figure 1 [2].

Furthermore, TDM can also be used to detect non-adherence early-on, which is thought to be one of the reasons of failure, relapse and resistant TB [3]. This is especially useful in China, where there is a high prevalence of non-adherence to $\mathrm{TB}$ treatment as well as resistant $\mathrm{TB}$, with $11 \%$ of all $\mathrm{TB}$ cases being MDR-TB $[1,15]$. Detecting non-adherence by plasma sampling is a more reliable and practical method 


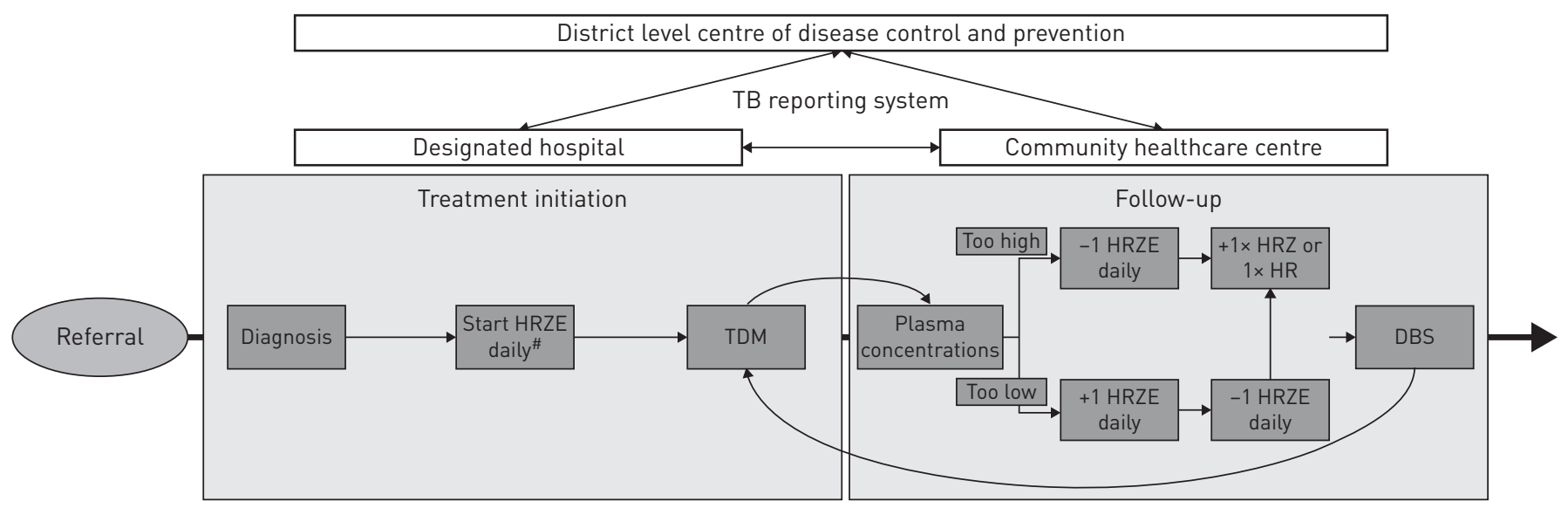

FIGURE 1 Example of fixed-dose combination (FDC) and therapeutic drug monitoring (TDM) in a programmatic setting in China. Following referral from all levels of health facilities, tuberculosis (TB) diagnosis is made by sputum smear and radiographic examination, followed by starting standard treatment with first-line anti-TB drugs isoniazid (H), rifampicin (R), pyrazinamide (Z) and ethambutol (E) in a TB-designated hospital/ clinic. The community health centre is appointed to commence directly observed treatment, short course. Blood sample collection is to be performed by the designated hospital/clinic after 2 weeks of treatment as part of TDM. After 2 weeks, plasma concentrations can be measured again by dried blood spot (DBS) sampling at the community health centre and if the plasma concentrations of isoniazid, rifampicin and/or pyrazinamide are too low, 1x HR or 1x HRZ is added. \#: the number of FDCs daily is based on the weight of the patient: $30-37 \mathrm{~kg}=2 ; 38-54 \mathrm{~kg}=3$; $55-70 \mathrm{~kg}=4$ and $\geqslant 71 \mathrm{~kg}=5$. HR=75/150 $\mathrm{mg} \mathrm{HRZ=80/120/250} \mathrm{mg,} \mathrm{HRZE=75/150/400/275} \mathrm{mg.}$

than urine testing, since drug plasma concentrations cannot be determined from urine. Additionally, since the rapid acetylator status is most common amongst the Asian population, TDM might also be of importance in this subpopulation in preventing suboptimal plasma-concentrations of isoniazid [6].

In settings with limited resources, we suggest to prioritise TDM for selected patients, such as patients with slow sputum conversion, risk of drug-drug interactions and comorbidities increasing the risk of low drug exposure (such as diabetes mellitus, HIV, gastro-intestinal disorders and other malabsorption diseases) [10].

We favour the use of FDC to simplify treatment and encourage the implementation of TDM. Recommendations in WHO and scientific communities' TB treatment guidelines about how and when to perform TDM should not only facilitate individual physicians in optimising treatment, but also facilitate policy makers in implementing TDM into National programmes.

@ERSpublications

Fixed-dose combination and therapeutic drug monitoring can be combined in programmatic treatment of tuberculosis http://ow.ly/sCtd302h0Ka

Marlanka A. Zuur ${ }^{1}$, Onno W. Akkerman ${ }^{2,3}$, Lina Davies Forsman ${ }^{4,5}$, Yi $\mathrm{Hu}^{6,7}$, Rongdong Zheng ${ }^{8}$, Judith Bruchfeld $^{4,5}$, Simon Tiberi ${ }^{9}$, Giovanni Battista Migliori ${ }^{10}$ and Jan-Willem C. Alffenaar ${ }^{1}$

${ }^{1}$ University of Groningen, University Medical Center Groningen, Dept of Clinical Pharmacy and Pharmacology, Groningen, The Netherlands. ${ }^{2}$ University of Groningen, University Medical Center Groningen, Tuberculosis Center Beatrixoord, Haren, The Netherlands. ${ }^{3}$ University of Groningen, University Medical Center Groningen, Dept of Pulmonary Diseases and Tuberculosis, Groningen, The Netherlands. ${ }^{4}$ Unit of Infectious Diseases, Dept of Medicine Solna, Karolinska Institutet, Stockholm, Sweden. ${ }^{5}$ Clinic of Infectious Diseases, Karolinska University Hospital Solna, Stockholm, Sweden. ${ }^{6}$ Dept of Epidemiology, School of Public Health, Fudan University, Shanghai, China. ${ }^{7}$ Key Laboratory of Public Health Safety (Fudan University), Ministry of Education, China. ${ }^{8}$ Dept of Tuberculosis Prevention, Xiamen City Center for Disease Control, Xiamen, China. ${ }^{9}$ Division of Infection, Barts Health NHS Trust, London, UK. ${ }^{10}$ World Health Organization Collaborating Centre for Tuberculosis and Lung Diseases, Fondazione S. Maugeri, Tradate, Italy.

Correspondence: Jan-Willem C. Alffenaar, University of Groningen, University Medical Center Groningen, Dept of Clinical Pharmacy and Pharmacology, Hanzeplein 1, 9713GZ Groningen, The Netherlands.

E-mail: j.w.c.alffenaar@umcg.nl

Received: April 272016 | Accepted after revision: July 06 2016 | First published online: Sept 012016

Conflict of interest: None declared.

\section{References}

1 World Health Organization. Global Tuberculosis Report 2015. http://apps.who.int/iris/bitstream/10665/191102/1/ 9789241565059_eng.pdf?ua=1 Date last accessed: November 23, 2015.

2 World Health Organization. Treatment of Tuberculosis Guidelines. Geneva, World Health Organization, 2010.

3 Mitchison DA. How drug resistance emerges as a result of poor compliance during short course chemotherapy for tuberculosis. Int J Tuberc Lung Dis 1998; 2: 10-15. 
4 Bangalore S, Kamalakkannan G, Parkar S, et al. Fixed-dose combinations improve medication compliance: a meta-analysis. Am J Med 2007; 120: 713-719.

5 Gallardo CR, Rigau Comas D, Valderrama Rodríguez A, et al. Fixed-dose combinations of drugs versus singledrug formulations for treating pulmonary tuberculosis. Cochrane Database Syst Rev 2016; 5: CD009913.

6 Pasipanodya JG, Srivastava S, Gumbo T. Meta-analysis of clinical studies supports the pharmacokinetic variability hypothesis for acquired drug resistance and failure of antituberculosis therapy. Clin Infect Dis 2012; 55: 169-177.

7 Goutelle S, Bourguignon L, Maire P, et al. The case for using higher doses of first line anti-tuberculosis drugs to optimize efficacy. Curr Pharm Des 2014; 20: 6191-6206.

8 Zuur MA, Bolhuis MS, Anthony R, et al. Current status and opportunities for therapeutic drug monitoring in the treatment of tuberculosis. Expert Opin Drug Metab Toxicol 2016; 12: 509-521.

9 Sun Q, Zhang Q, Gu J, et al. Prevalence, risk factors, management, and treatment outcomes of first-line antituberculous drug-induced liver injury: a prospective cohort study. Pharmacoepidemiol Drug Saf 2016; 25: 908-917.

10 van der Burgt EP, Sturkenboom MG, Bolhuis MS, et al. End TB with precision treatment! Eur Respir J 2016; 47: $680-682$.

11 Ghimire S, Bolhuis MS, Sturkenboom MG, et al. Incorporating therapeutic drug monitoring into the World Health Organization hierarchy of tuberculosis diagnostics. Eur Respir J 2016; 47: 1867-1869.

12 Sotgiu G, Alffenaar JW, Centis R, et al. Therapeutic drug monitoring: how to improve drug dosage and patient safety in tuberculosis treatment. Int J Infect Dis 2015; 32: 101-104.

13 Pasipanodya JG, McIlleron H, Burger A, et al. Serum drug concentrations predictive of pulmonary tuberculosis outcomes. J Infect Dis 2013; 208: 1464-1473.

14 Pasipanodya JG, Gumbo T. Clinical and toxicodynamic evidence that high-dose pyrazinamide is not more hepatotoxic than the low doses currently used. Antimicrob Agents Chemother 2010; 54: 2847-2854.

15 Lei X, Huang K, Liu Q, et al. Are tuberculosis patients adherent to prescribed treatments in China? Results of a prospective cohort study. Infect Dis Poverty 2016; 5: 38.

\section{Tuberculosis in London: the convergence of clinical and social complexity}

\section{To the Editor:}

In large European cities, the tuberculosis (TB) epidemic is characteristically concentrated in vulnerable and under-served populations [1]. London has the highest number and annual incidence of TB in Europe and implemented routine surveillance on homelessness, drug and alcohol misuse and imprisonment among TB patients in 2009 [2]. This paper describes the clinical, public health and epidemiological characteristics of $\mathrm{TB}$ cases and the public health impact of social risk factors including risk of infectiousness, onward transmission, poor treatment adherence and drug resistance.

We analysed a cohort of adult London TB patients (2009-2012) including clinical and laboratory surveillance information. This was improved by matching against the Find\&Treat team's database, who support TB patients across London with complex social needs [3]. Homelessness, imprisonment, drug and alcohol misuse were defined as per national guidance [4]. Multi-drug resistant (MDR) TB was defined as per the World Health Organization (WHO), and poor treatment outcome was defined as not completing treatment within 12 months for rifampicin-sensitive patients, or within 24 months for rifampicin-resistant patients [5]. Recent migrants were defined as entering the UK less than 2 years before diagnosis. United Nations world region of birth was amended to a TB surveillance classification. Ethical approval was not required as this study was based on routine surveillance data held by Public Health England. Public Health England has Health Research Authority approval to hold and analyse national surveillance data for public health purposes.

Risk factors were identified for smear-positive pulmonary disease; isoniazid and MDR (restricted to culture-confirmed cases); non-adherence to treatment; and poor treatment outcomes (restricted to individuals notified 2009-2011). Univariable analysis generated odds ratios, with 95\% confidence intervals and Chi-squared test for significance. Multivariable logistic regression was used to generate adjusted odds ratios, built using likelihood ratio tests. Variables were retained in the final model if they improved the fit of the model $(\mathrm{p}<0.05)$ or confounded a different exposure. Potential interactions were investigated based on a priori knowledge. Data were analysed using Stata 12 (StataCorp LP, College Station, TX, USA).

Of the cohort of 12908 adult TB cases, 1321 (10\%) had one or more social risk factor: homelessness (550 (4\%)), imprisonment (349 (3\%)), drug (436 (3\%)) or alcohol misuse $(581(5 \%))$. Cases with social risk factors were more often male $(79 \%$ versus $55 \%$; $\mathrm{p}<0.001)$ UK born $(29 \%$ versus $12 \%$; $\mathrm{p}<0.001)$ white $(25 \%$ versus $9 \% ; \mathrm{p}<0.001)$ or black Caribbean $(7 \%$ versus $3 \% ; \mathrm{p}<0.001)$. Multiple factors were common (393 patients, 30\% reported two or more). 